Chapter 9: Flares and star-planet interaction 


\title{
Magnetism and activity of planet hosting stars
}

\author{
Jason T. Wright ${ }^{1}$ and Brendan P. Miller ${ }^{2}$ \\ ${ }^{1}$ Center for Exoplanets and Habitable Worlds \\ and \\ Department of Astronomy and Astrophysics \\ 525 Davey Laboratory, The Pennsylvania State University, University Park, PA 16802 \\ email: astrowright@gmail.com \\ ${ }^{2}$ Department of Chemistry and Physical Sciences, The College of St. Scholastica, Duluth, MN \\ 55811
}

\begin{abstract}
The magnetic activity levels of planet host stars may differ from that of stars not known to host planets in several ways. Hot Jupiters may induce activity in their hosts through magnetic interactions, or through tidal interactions by affecting their host's rotation or convection. Measurements of photospheric, chromospheric, or coronal activity might then be abnormally high or low compared to control stars that do not host hot Jupiters, or might be modulated at the planet's orbital period. Such detections are complicated by the small amplitude of the expected signal, by the fact that the signals may be transient, and by the difficulty of constructing control samples due to exoplanet detection biases and the uncertainty of field star ages. We review these issues, and discuss avenues for future progress in the field.
\end{abstract}

Keywords. stars:activity, stars:chromospheres, stars:magnetic fields stars:planetary systems, stars:rotation, stars:spots

\section{Chromospheric Activity as a Confounder In Radial Velocity Searches for Planets}

\subsection{Jitter and RV-activity Correlations}

Studies of stellar magnetic activity of potential planet host stars are almost as old as radial velocity planet searches. Campbell et al. (1991) reported the discovery of a correlation between differential precise radial velocities and magnetic activity level among active stars. Walker et al. (1995) showed that the phenomenon was usually not important in their sample of 21 dwarf stars, but did see strong correlations in 10 years of precise $\kappa^{1}$ Ceti radial velocities. This phenomenon is now known to be ubiquitous, at a low level (see, for instance, Lovis et al. (2011), Dumusque et al. (2012)).

Because the correlation between activity level and radial velocity is imperfect, and in some cases absent despite large variance in both quantities, active stars have generally been avoided in radial velocity planet searchers. Wright et al. (2004) performed comprehensive analysis of the chromospheric activity level of stars in the California Planet Survey at Lick and Keck Observatories, including both overall levels and time series from every exposure used for radial velocity work, and Isaacson \& Fischer (2010) provided an additional six years of measurements for Keck observations. The cadence is a few observations per year for most stars, with higher cadences for bright and multiplanet host stars.

Wright (2004) used these data in an attempt to quantify the amount of radial velocity "jitter" to be expected from stars of a given temperature, evolutionary state, and level of chromospheric activity. The variance among stars with similar paramaters in the sample is 
large, but in general this jitter increases with activity level, degree of evolution (measured as height above the $V$-band Main Sequence, $\Delta M_{V}$ ), and emission in the core of the Ca II $\mathrm{H} \& \mathrm{~K}$ lines.

A second way that stellar magnetic activity confounds radial velocity searches for planets is by rotational modulation of starspots. Spots on the approaching limb of a star can suppress the most blueshifted component of a star's light, producing a spurious redshift measurement. Spots of sufficient size to cause a very large such shift will also be apparent photometrically as stellar brightness and color variations with a $\pi / 2$ phase shift with respect to the radial velocities, as in the case of HD 166435 (Queloz et al. 2001). In this manifestation, the chromospheric activity enhancement near the spot can sometimes be also seen with a $-\pi / 2$ phase shift from the spurious Doppler signal. More complex modeling of this phenomenon, which accounts for additional effects such as those from plages and the inhibition of convective blueshift, is also possible, as in the case, for instance, of $\mathrm{FF}^{\prime}$ (Aigrain et al. 2012) and SOAP (Boisse et al. 2013; Dumusque et al. 2014).

\subsection{Stellar Cycles and Long Period Planets}

Dravins (1985) predicted spurious radial velocity variations due to changes in patterns of surface convection during a stellar cycle. He recommended tracking precise radial velocities of Solar lines of different strength, excitation potential, and wavelength to probe these effects. Deming et al. (1987) reported precise radial velocities of the Sun in the $\Delta V=2$ transitions of ${ }^{12} \mathrm{C}^{16} \mathrm{O}$ at $2.3 \mu$ in three epochs spanning four years. They reported that daily variations were below $3 \mathrm{~m} / \mathrm{s}$, but they inferred a large shift of -30 $\mathrm{m} / \mathrm{s}$ over that span, which they attributed to the solar cycle. They inferred that there would therefore be a lower limit to the mass of planets that could be detected around Sun-like stars due to this confounding effect. $\dagger$ In spite of these predictions and apparent demonstrations that stellar cycles would be a major problem for radial velocity planet searches, there have been few manifestations of this problem. The matter was addressed directly by Wright et al. (2008), who found a correlation among the radial velocities, activity levels, and brightness of the star HD 154345. They noted that a near spectral twin, $\sigma$ Draconis (HD 185144) had a stronger stellar cycle and higher overall activity level, and yet serves as a quintessential precise radial velocity stable star for groups around the world. The lack of an obvious correlation between radial velocity and activity in $\sigma$ Draconis (and indeed most cycling stars in the California Planet Survey) and the strength of the RV signal in HD 154345 (over $10 \mathrm{~m} / \mathrm{s}$ ) led those authors to conclude that the correlation was an inevitable coincidence and that HD 154345 is orbited by a Jupiter analog (indeed, the first good one discovered). Similarly, Santos et al. (2010) found no evidence for RV-activity correlations due to cycles in a sample of early $\mathrm{K}$ dwarfs.

Subsequent observations have cast the conclusions of Wright et al. (2008) and Santos et al. (2010) into doubt, however, at least for some stars. Moutou et al. (2011) identified two $\mathrm{K}$ stars in their survey with activity-RV correlations with amplitudes $>10 \mathrm{~m} / \mathrm{s}$ and timescales of years (and confirmed via line bisectors that the RVs were indeed spurious). Carolo et al. (2014) showed a similar correlation in an active K0 star with an amplitude of $\sim 15 \mathrm{~m} / \mathrm{s}$, as did Robertson et al. (2013) for a K8/M0 star (amplitude $\sim 10 \mathrm{~m} / \mathrm{s}$ ). Similarly, Dumusque et al. (2012) showed a signal with a $\sim 5 \mathrm{~m} / \mathrm{s}$ amplitude in the K1 star $\alpha$ Cen B, and Gomes da Silva et al. (2012) found a similar low-level $(<5 \mathrm{~m} / \mathrm{s})$ correlation in a sample of M dwarfs. Indeed, subsequent observations of HD 154345 have

$\dagger$ Although, since stellar cycles can be monitored, in practice such an effect can be detected and removed, and so would not really present an insurmountable hurdle to planet detection, even if it were pervasive. 

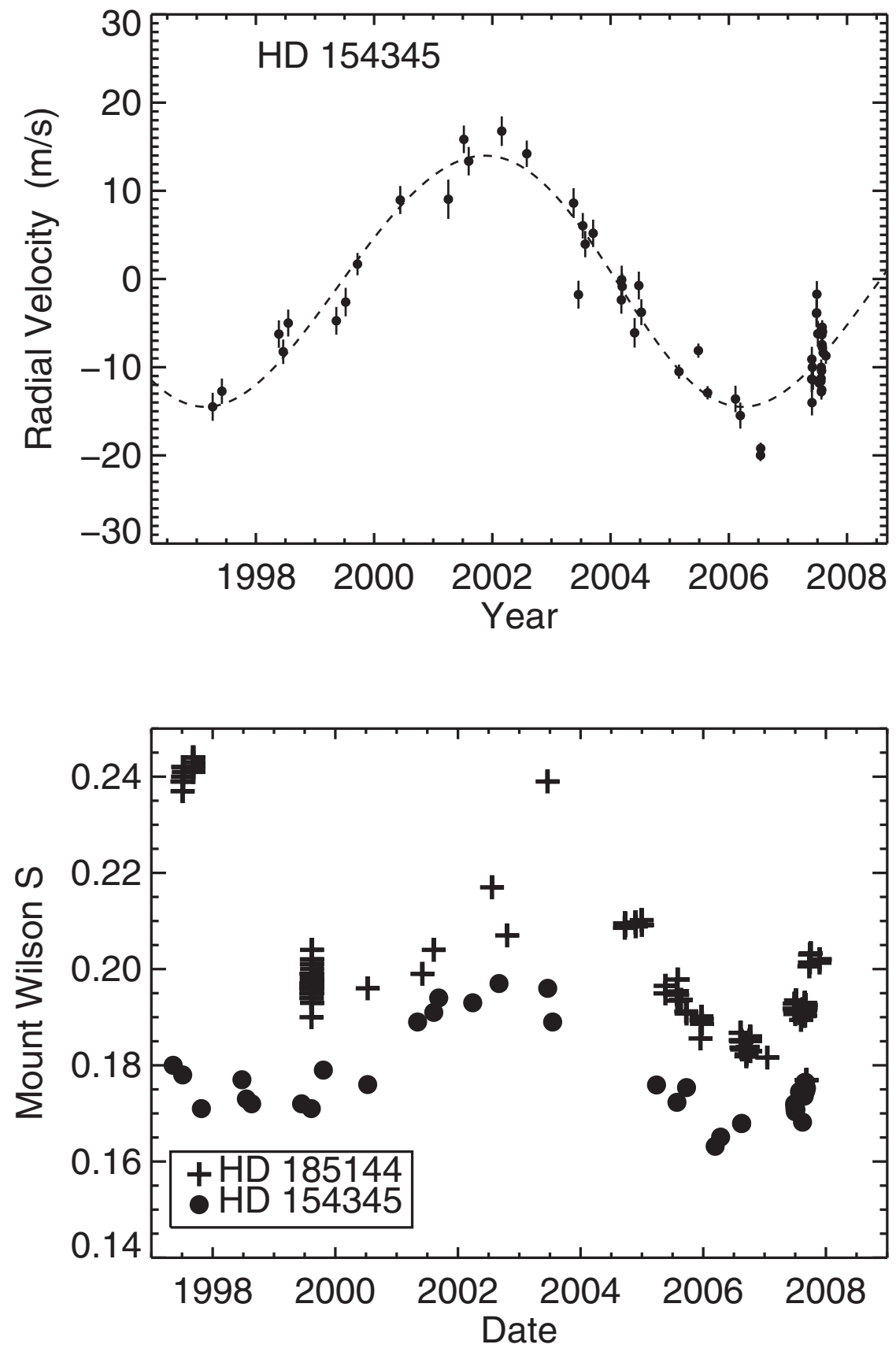

Figure 1. Figures 1 (top) \& 2 (bottom) from Wright et al. (2008), illustrating the strange case of HD 154345, purportedly the first good Jupiter analog. The apparently strong and clean RV signal is clearly well correlated with the Mount Wilson $S$ index, a measurement of chromospheric activity (circles, bottom). HD 185144 (= $\sigma$ Draconis, crosses, bottom) is more active and has a stronger activity cycle, but is a radial velocity standard star. 
made the coincidence in that system even stronger, casting doubt on the reality of the planet.

But the broader point made by Wright et al. (2008) and Santos et al. (2010) (and, implicitly, by Gomes da Silva et al. (2012)) still stands: most stars do not show large RV variations due to activity cycles, though many may at a low level below that induced by Jupiter-mass planets. The question of why a loud minority of stars do show strong correlations that can be misinterpreted as giant planets remains open.

The best comprehensive analysis to date of the effect is the manuscript of Lovis et al. (2011), who found that RV-activity correlations due to stellar cycles are more common in hotter stars and almost absent in cooler stars (a finding contradicted by many of the citations above), and found average RV-activity correlation coefficients as a function of effective temperature and metallicity (although, as we have seen, there must be a large amount of scatter about this relation).

\section{Star-Planet Interactions}

\subsection{Modes of Proposed Star-Planet Interactions}

Might the physical connection between activity and RV variation go the other way? Jose (1965) suggested, motivated by the similarity of the Sun's $\sim 11$ year activity cycle to the combined orbital action of Jupiter and Saturn, that the solar cycle is in some way caused by its planets; Wilson et al. (2008) developed this idea further, and Perryman \& SchulzeHartung (2011) argued that this hypothesis could be tested in exoplanetary systems. While the idea has an certain appealing counter-intuitiveness to it, Shirley (2006) has shown that there is really no physical mechanism that could plausibly produce such coupling, $\dagger$ (although Abreu et al. (2012) would beg to differ).

Close-in planets, however, could very plausibly interact magnetically and tidally with their host stars in detectable ways. Indeed, an exciting consequence of the stellar magnetic field is how it might interact with and so reveal the strength of close-in planetary magnetic fields.

Cuntz et al. (2000) gave initial estimates of the interaction strengths of magnetic interactions of stars and their planets, and Rubenstein \& Schaefer (2000) suggested the effects of close-in giant planets might be so large as to be responsible for superflares seen in old FGK stars. Much work has been done to refine these early estimates. For instance, Cohen et al. (2009) performed time-dependent MHD simulations, and found that there should be detectable enhancements in X-ray emission and chromospheric activity (see also Lanza 2008, 2009; Saur et al. 2013; Cohen et al. 2011).

These effects fall into two broad observational classes. The first is that a close-in planet might "tickle" the star's field, causing orbital energy to be dissipated at the footprint of the field lines in the chromosphere, resulting in a chromospheric "hot spot," observed to be modulated on an orbital timescale. The second is that, through a variety of mechanisms, a close-in planet might alter the overall level of chromospheric activity.

$\dagger$ Specifically, Jose (1965) and Wilson et al. (2008) suggest that the Sun's global field strength is dictated by its physical position with respect to the Solar System barycenter. But by the Equivalence Principle, the gravitational pull of the planets cannot affect the Sun's internal motions any more than an astronaut in a small ship can use accelerometers to determine whether they are in orbit or deep space. Any viable mechanism must therefore invoke either gravitational tides (which are minuscule here) or purely electromagnetic effects. 


\subsection{Orbitally Modulated Activity}

The first of these effects can be tested in a few nights' observing time via a time series of strengths of chromospheric emission lines, such as the Ca II H \& K lines (Saar \& Cuntz 2001). Shkolnik et al. (2003) claimed the first detection of this effect as a modulated line strength in the star HD 179949 due to its hot Jupiter companion; the effect was apparently persistent over three observing runs in 2001 and 2002 and the authors later claimed to see similar effects in other stars.

Variation in chromospheric line strengths are expected as magnetically active regions rotate into and out of view on the star, but Shkolnik et al. (2003) found that they phased better with the orbital period of the planet than the rotational period of the star (although Miller et al. (2012) showed that with poor phase coverage such distinctions can be difficult or impossible to make).

Shkolnik et al. (2005) appeared to confirm the result, and to find a similar effect in the $v$ And system. Shkolnik et al. (2008) found the detections of these signals difficult to reproduce, leading them to speculate that the effect has an "on/off nature" (see Figure 2). Indeed, Poppenhaeger et al. (2011) found no variability in the $v$ And system at the orbital period of its close-in planet in Ca II H \& $\mathrm{K}$ or in X-rays, nor did Scandariato et al. (2013) in their observing campaign on HD 179949. The difficulty in confirming this mode of interaction since its putative discovery casts doubt on the interpretation that the variations were necessarily due to planetary perturbations.

\subsection{Enhancement of Overall Activity}

Detection of an overall enhancement (or decrease) in activity levels is more difficult because in general one must know the "correct" level for the star, which might have a cycle. Most work on this topic has thus been done statistically, to determine if stars with hot Jupiters appear unusually active. Such work requires careful construction of a control sample, since there are many observational selection effects in searches for hot Jupiters that can confound such an analysis (Poppenhaeger \& Schmitt 2011b).

Kashyap et al. (2008) performed an analysis of X-ray emission from a sample of planethosting stars, searching for correlations with orbital distance (since the strength of the effect should decrease with increasing orbital distance). They found that stars with closein planets are more X-ray active by a factor of 4 than those with distant planets, but this conclusion is subject to several difficulties, including non-planets orbiting very active stars (e.g. they included the brown dwarf candidate Cha $\mathrm{H} \alpha 8 \mathrm{~B}$, whose host star is active primarily because it is 3 Myr old (Joergens et al. 2010)) and observational biases (transit surveys sensitive to close-in planets can sample more active stars, while long-period planets are preferentially discovered around inactive stars favored by radialvelocity methods). They attempt to correct for sample bias and estimate a planet-induced activity enhancement by a factor of 2. However, Poppenhaeger et al. (2010) performed a more careful analysis with a more sensitive set of X-ray data and found no evidence for elevated activity among hosts of hot Jupiters.

Scharf (2010) tentatively identified a relation between stellar $L_{X}$ and planet mass for close-in planets (that is not present for more distant planets) in ROSAT All-Sky Survey data. Poppenhaeger \& Schmitt (2011a) showed that this correlation did not survive the addition of more sensitive XMM-Newton data, and that the correlations that had been seen in the past were likely due to selection effects in the planet-search process.

But more carefully constructed samples have hinted at a more subtle role for hot Jupiters to play. Poppenhaeger \& Wolk (2014) examined coronal X-rays from the components of wide binary stars in which one star hosts a hot Jupiter, using its companion as a negative control, and found that the Jupiter hosts do seem to be more active than 

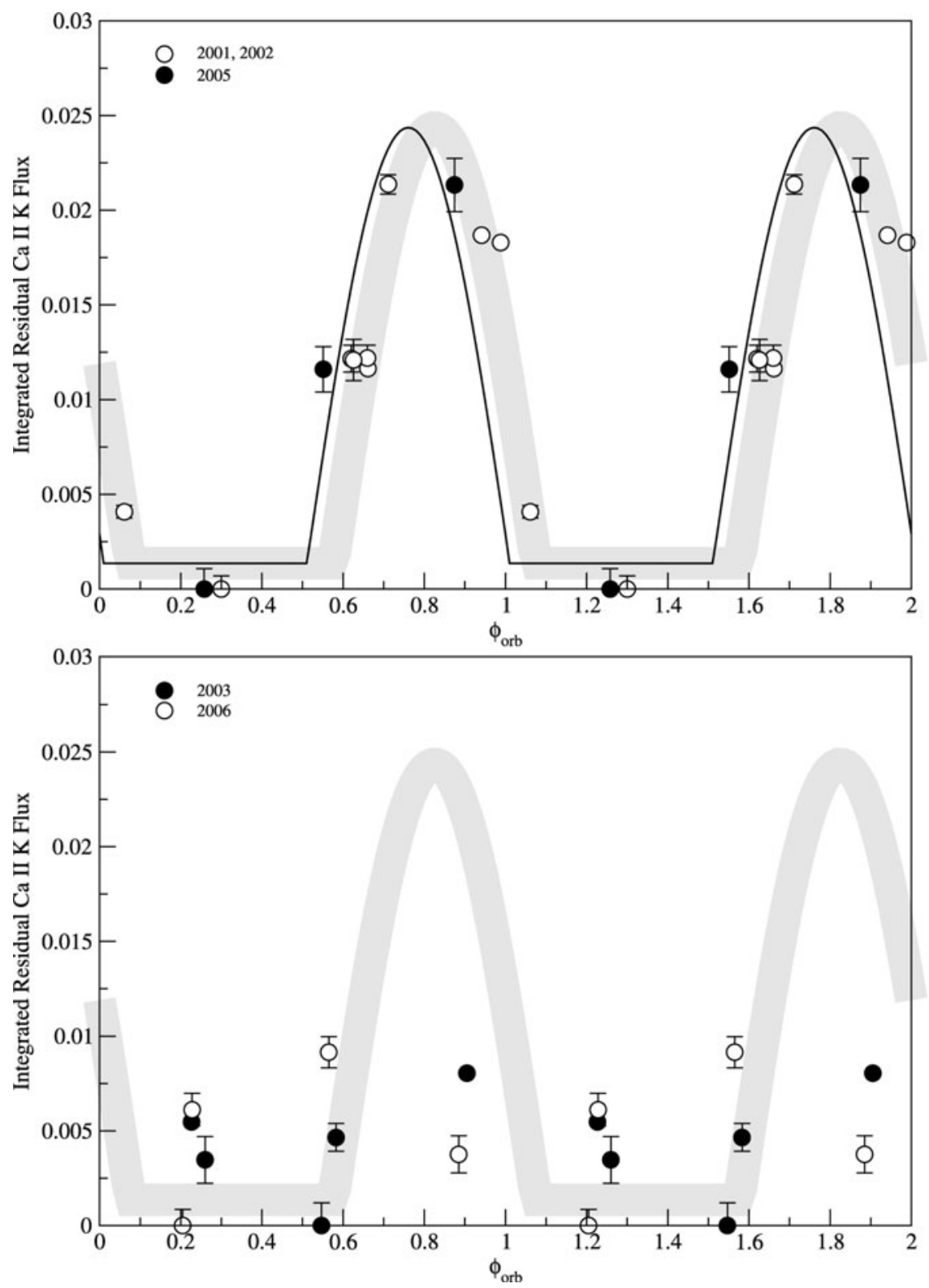

Figure 2. Figures 6 (top) \& 7 (bottom) from Shkolnik et al. (2008) showing the "on-off" nature of star-planet interactions in HD 179949. Note that each data point appears twice in these plots because phase runs from zero to 2 to emphasize the purportedly periodic nature of the signal. On the top, the "on" epochs, which have a slightly different best-fit phase in the 2001-2 epoch (thick gray line) than in the 2005 epoch (black line). On the bottom, the "off" epochs where no effect is seen.

their coeval sibling. Miller et al. (2015) performed a comprehensive and careful assessment of X-ray data of exoplanet hosts, seeking correlations in coronal X-ray strength with plausible metrics of star-planet magnetic interaction $\left(M_{p} / a^{2}, 1 / a\right)$. They found no significant elevation in a sample of solar analogs (Figure 3), but did find a significant correlation driven by a few very massive, very close-in exoplanets orbiting X-ray bright 


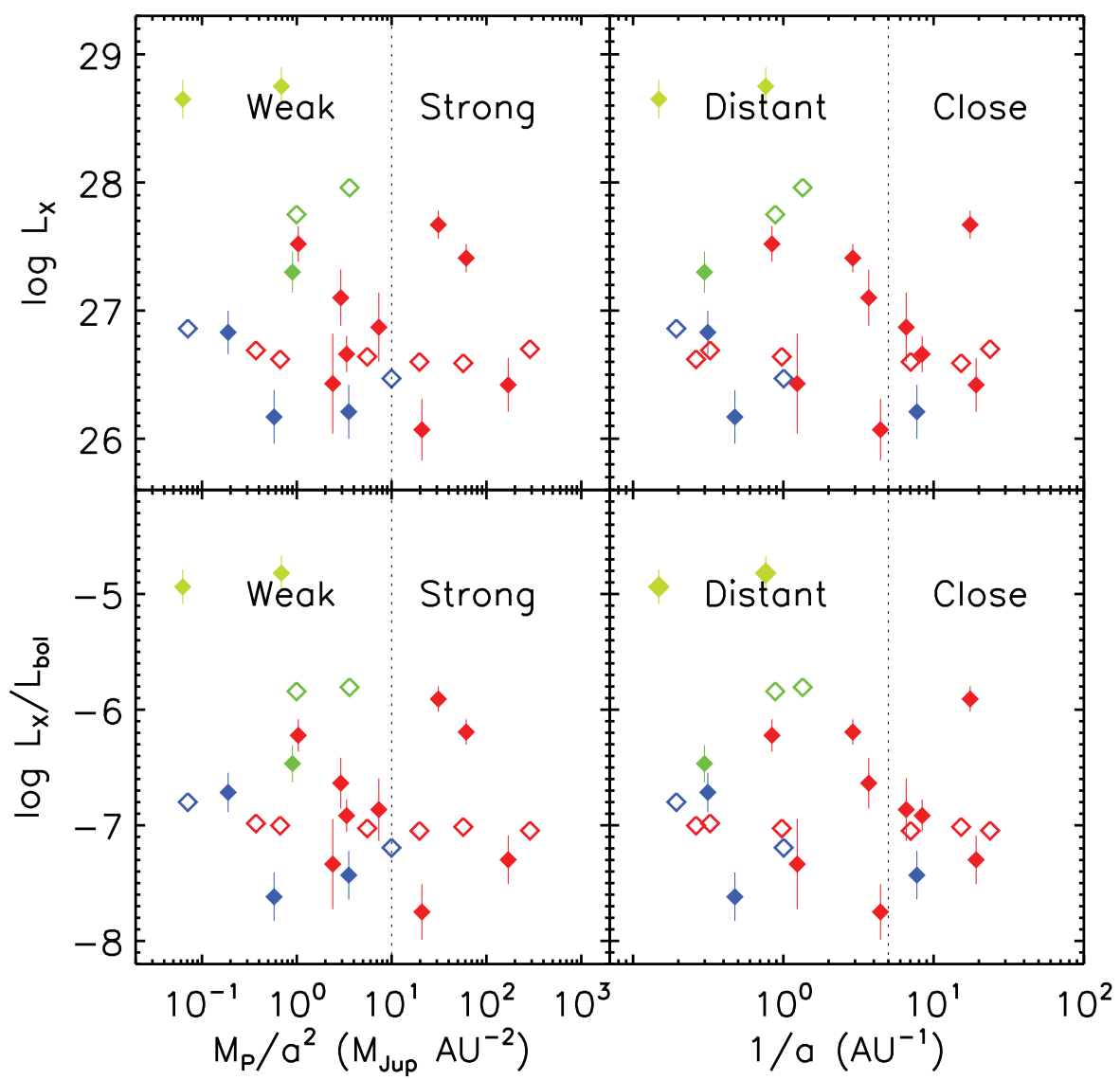

Figure 3. Figure 4 of Miller et al. (2015), showing the distribution of coronal activity vs. plausible scalings for planet-star interaction strength for solar analogs in their sample. Open symbols represent upper limits. Chandra data are red, XMM-Newton data are blue, and ROSAT data are green. Miller et al. (2015) found no significant increase in X-ray emission among the stars most likely to show elevated activity due to star-planet interactions. Light green points were excluded from their analysis as being "atypically active."

stars (Figure 4). These stars' activity levels were consistent with their chromospheric emission and rotation rates, and so did not appear to be elevated because of magnetic interactions with the planet.

\subsection{An Indirect Mode For Magnetic Activity Enhancement Via Star-Planet Interactions}

Both Poppenhaeger \& Wolk (2014) and Miller et al. (2015) argued that the source of the elevated activity levels is consistent with the stars having been spun up by their massive, close-in planets. In this scenario, the normal spin-down of stars with age is slowed or halted by tidal interactions with their planets, and so their dynamos remain strong late into the stars' lives. Such action is instructive for determining the factors contributing to tidal coupling between stars and planets, but dampens hopes for using stellar magnetic fields to probe exoplanetary magnetic fields. 


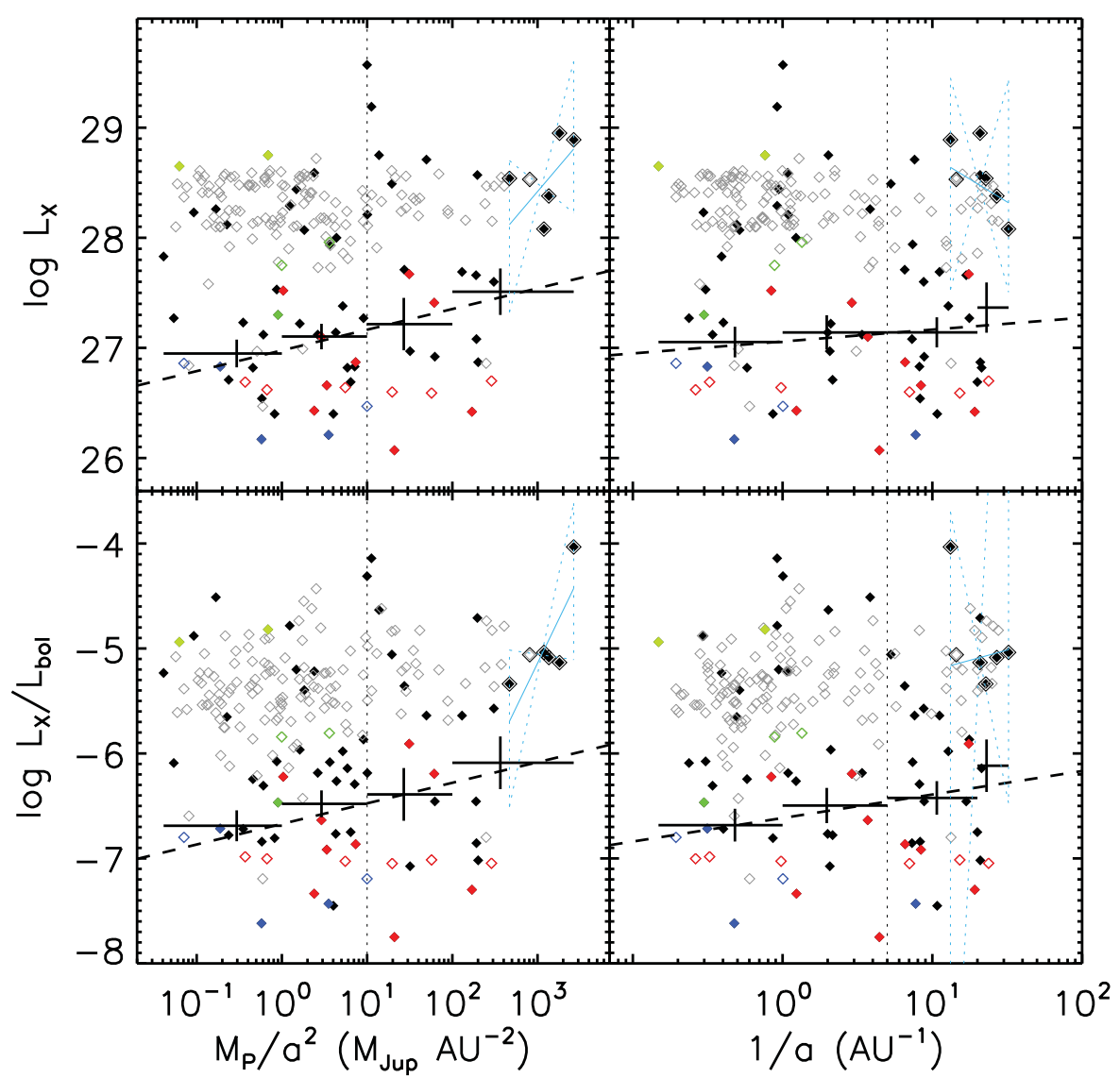

Figure 4. Figure 7 of Miller et al. (2015), showing the distribution of coronal activity vs. plausible scalings for planet-star interaction strength for their entire sample, with the same color scheme as Figure 3 for the Solar analogs, and other stars in black. The double diamond symbols (which have a fit shown in cyan) are "extreme" systems which drive the weak correlation shown by the black dashed line.

\section{WASP-18 as Case Study}

\subsection{A Search in the Strongest Candidate for SPI Comes Up Empty}

WASP-18b (Hellier et al. 2009; Southworth et al. 2009) is an ultra-short period transiting hot Jupiter with $M_{p}=10 \mathrm{M}_{\mathrm{Jup}}$ orbiting an F6 star. The brightness of the host star $(V=9.3)$ and the mass and orbital distance of the planet make the WASP-18 system one of the best candidates for observations of star planet interactions.

Strangely, however, WASP-18 is unusually inactive, both chromospherically (Knutson et al. 2010; Fossati et al. 2014) and coronally (Miller et al. 2012; Pillitteri et al. 2014). It has $\log R_{\mathrm{HK}}^{\prime}=-5.15$, which is extraordinarily low for a main sequence star, and is more typical of subgiants (Wright 2004), despite the star having a rotation period (5.6 days) ordinary for its spectral type and age.

This low level of activity frustrated the attempts of Miller et al. (2012) and Pillitteri et al. (2014) to monitor the system for orbitally modulated activity in X-rays; it was not detected in $50 \mathrm{ks}$ of Swift time or in $87 \mathrm{ks}$ of Chandra time. If this system does not show enhanced activity due to star-planet interactions, other, less favorable systems might not be expected to show anything detectable. 


\subsection{A Different Manifestation of Planetary Influence?}

The solution to the puzzle of the low activity levels of WASP-18 may be the extraordinarily large and close-in planet orbiting it. One possibility, mentioned by Miller et al. (2012), is that planetary tides have in some way disrupted the dynamo action of the star (see also Wolk, Pillitteri, and Poppenhaeger, in these proceedings).

A second suggestion, by Fossati et al. (2013), is that the chromospheric measurements of WASP-18 are contaminated by absorption from intervening gas escaping from the planet (Fossati et al. (2014) later ruled out ISM absorption). This mechanism has been suggested by Lanza (2014) and Fossati et al. (2015) to explain the correlation, first identified by Hartman (2010), that low surface gravity hot Jupiters tend to orbit stars that appear less active. The very high surface gravity of WASP-18 would seem to cut against such a possibility in this instance, but the concern is apparently warranted for WASP-12 (Fossati et al. 2013), and presumably other systems as wall. At any rate, Pillitteri et al. (2014) ruled this mechanism out for WASP-18, since the star is also unusually faint in hard X-rays, which should be relatively unattenuated by a gas cloud.

Pillitteri et al. (2014) note that the star has significant lithium, more than expected even for a Hyades-aged star. Pillitteri et al. (2014) combine the mystery of the star's high lithium abundance and low activity with a tidal strength calculation, and infer that WASP-18 $b$ has indeed disrupted its host star's dynamo via tidal suppression of convection in the shallow convective zone - an effect that also served to frustrate lithium burning at the base of the convective zone. This hypothesis would gain support if the combination of unusually low activity and unusually high lithium abundance is common in similar stars with very close, massive companions (i.e. stars, brown dwarfs, or very massive planets). Such an observation would cut against the general trend of late F stars hosting hot Jupiters to have lower lithium abundances than stars without hot Jupiters (Delgado Mena et al. 2015).

\section{References}

Abreu, J. A., Beer, J., Ferriz-Mas, A., McCracken, K. G., \& Steinhilber, F. 2012, A\& A, 548, A 88

Aigrain, S., Pont, F., \& Zucker, S. 2012, MNRAS, 419, 3147

Boisse, I., Bonfils, X., Santos, N. C., \& Figueira, P. 2013, in Astronomical Society of the Pacific Conference Series, Vol. 472, New Quests in Stellar Astrophysics III: A Panchromatic View of Solar-Like Stars, With and Without Planets, ed. M. Chavez, E. Bertone, O. Vega, \& V. De la Luz, 259

Campbell, B., Yang, S., Irwin, A. W., \& Walker, G. A. H. 1991, in Lecture Notes in Physics, Berlin Springer Verlag, Vol. 390, Bioastronomy: The Search for Extraterrestial Life - The Exploration Broadens, ed. J. Heidmann \& M. J. Klein, 19-20

Carolo, E., Desidera, S., Gratton, R., et al. 2014, A\&A, 567, A48

Cohen, O., Drake, J. J., Kashyap, V. L., et al. 2009, ApJ, 704, L85

Cohen, O., Kashyap, V. L., Drake, J. J., et al. 2011, ApJ, 733, 67

Cuntz, M., Saar, S. H., \& Musielak, Z. E. 2000, ApJ, 533, L151

Delgado Mena, E., Bertrán de Lis, S., Adibekyan, V. Z., et al. 2015, A\&GA, 576, A69

Deming, D., Espenak, F., Jennings, D. E., Brault, J. W., \& Wagner, J. 1987, ApJ, 316, 771

Dravins, D. 1985, in Stellar Radial Velocities, ed. A. G. D. Philip \& D. W. Latham, 311-320

Dumusque, X., Boisse, I., \& Santos, N. C. 2014, ApJ, 796, 132

Dumusque, X., Pepe, F., Lovis, C., et al. 2012, Nature, 491, 207

Fossati, L., Ayres, T. R., Haswell, C. A., et al. 2013, ApJ, 766, L20

-. 2014, ApESSS, 354, 21

Fossati, L., Ingrassia, S., \& Lanza, A. F. 2015, ApJ, 812, L35

Gomes da Silva, J., Santos, N. C., Bonfils, X., et al. 2012, A\&sA, 541, A9 
Hartman, J. D. 2010, ApJ, 717, L138

Hellier, C., Anderson, D. R., Collier Cameron, A., et al. 2009, Nature, 460, 1098

Isaacson, H., \& Fischer, D. 2010, ApJ, 725, 875

Joergens, V., Müller, A., \& Reffert, S. 2010, A\& A, 521, A24

Jose, P. D. 1965, AJ, 70, 193

Kashyap, V. L., Drake, J. J., \& Saar, S. H. 2008, ApJ, 687, 1339

Knutson, H. A., Howard, A. W., \& Isaacson, H. 2010, ApJ, 720, 1569

Lanza, A. F. 2008, $A \mathscr{6} A$, 487, 1163

-. 2009, $A \& A, 505,339$

-. 2014, $A \mathscr{G} A, 572, \mathrm{~L} 6$

Lovis, C., Dumusque, X., Santos, N. C., et al. 2011, A\&SA, submitted, arXiv:1107.5325, arXiv:1107.5325

Miller, B. P., Gallo, E., Wright, J. T., \& Dupree, A. K. 2012, ApJ, 754, 137

Miller, B. P., Gallo, E., Wright, J. T., \& Pearson, E. G. 2015, ApJ, 799, 163

Moutou, C., Mayor, M., Lo Curto, G., et al. 2011, A\&A, 527, A63

Perryman, M. A. C., \& Schulze-Hartung, T. 2011, A\& $A$, 525, A65

Pillitteri, I., Wolk, S. J., Sciortino, S., \& Antoci, V. 2014, A\& A, 567, A128

Poppenhaeger, K., Lenz, L. F., Reiners, A., Schmitt, J. H. M. M., \& Shkolnik, E. 2011, A\&A, 528, A 58

Poppenhaeger, K., Robrade, J., \& Schmitt, J. H. M. M. 2010, A\&A, 515, A98

Poppenhaeger, K., \& Schmitt, J. H. M. M. 2011a, ApJ, 735, 59

-. 2011b, Astronomische Nachrichten, 332, 1052

Poppenhaeger, K., \& Wolk, S. J. 2014, A\&A, 565, L1

Queloz, D., Henry, G. W., Sivan, J. P., et al. 2001, A\&A, 379, 279

Robertson, P., Endl, M., Cochran, W. D., MacQueen, P. J., \& Boss, A. P. 2013, ApJ, 774, 147

Rubenstein, E. P., \& Schaefer, B. E. 2000, ApJ, 529, 1031

Saar, S. H., \& Cuntz, M. 2001, MNRAS, 325, 55

Santos, N. C., Gomes da Silva, J., Lovis, C., \& Melo, C. 2010, A\&AA, 511, A54

Saur, J., Grambusch, T., Duling, S., Neubauer, F. M., \& Simon, S. 2013, A\&AA, 552, A119

Scandariato, G., Maggio, A., Lanza, A. F., et al. 2013, A\& $A$, 552, A7

Scharf, C. A. 2010, ApJ, 722, 1547

Shirley, J. H. 2006, MNRAS, 368, 280

Shkolnik, E., Bohlender, D. A., Walker, G. A. H., \& Collier Cameron, A. 2008, ApJ, 676, 628

Shkolnik, E., Walker, G. A. H., \& Bohlender, D. A. 2003, ApJ, 597, 1092

Shkolnik, E., Walker, G. A. H., Bohlender, D. A., Gu, P.-G., \& Kürster, M. 2005, ApJ, 622, 1075

Southworth, J., Hinse, T. C., Dominik, M., et al. 2009, ApJ, 707, 167

Walker, G. A. H., Walker, A. R., Irwin, A. W., et al. 1995, Icarus, 116, 359

Wilson, I. R. G., Carter, B. D., \& Waite, I. A. 2008, PASA, 25, 85

Wright, J. T. 2004, AJ, 128, 1273

Wright, J. T., Marcy, G. W., Butler, R. P., \& Vogt, S. S. 2004, ApJS, 152, 261

Wright, J. T., Marcy, G. W., Butler, R. P., et al. 2008, ApJ, 683, L63 